\title{
Physics in The Netherlands
}

\section{H. Eggen}

\author{
FOM, Utrecht, The Netherlands
}

English contains a number of expressions where the word "Dutch" has a negative connotation. Wars between the Dutch and the English in the 17th century must be responsible for this. However, there is one definite exception: Dutch physics, which in the United States is synonymous with statistical physics reflecting the impact of intellectual giants such as Lorentz, Kamerlingh Onnes, Van der Waals and Zeeman, all of whom have laboratories named after themselves.

Statistical physics remains one of the strong points of physics research in The Netherlands. Examples include the work on light induced drift (Woerdman, University of Leiden — see box: The Optical Piston) and on atomic hydrogen (Walraven, University of Amsterdam), simulations of crystals (Frenkel and Ernst, University of Utrecht) and the widely acclaimed work on the physics of colloids by Vrij (also at the University of Utrecht).

One of the reasons for the success in The Netherlands of experimental work in "small science" fields like statistical physics is the fact that Dutch instrument makers are among the best in the world. They form part of a long tradition going back to the likes of Van Leeuwenhoek, Van Musschenbroek and Stevin.

Atomic physics and quantum electronics are two other fields of small science to which Dutch physicists have contributed greatly. Among the many possible examples of topics that have yielded remarkable results we have the study of the localization of light (Lagendijk, University of Amsterdam - see box). One should also not forget measurements of the wave function of electrons (see box: Electron Wave Function Measured) and of the onset of melting in solids (H.B. Van Linden van den Heuvell and colleagues and Van der Veen and Frenken respectively, at the FOM - Institute for Atomic and Molecular Physics, Amsterdam).

Important results have also been reported by groups working in condensed matter physics including, for instance, quantized conductivity between point contacts (discovered by Van Wees and coworkers, University of Delft and Philips, Eindhoven), the production of sandwiched high $T_{c}$ superconducting films (Rogalla, University of Enschede) and theoretical work on these new ceramic superconductors (Sawatzky, University of Groningen).
But Dutch physicists have also been working for a long time in the fields of high energy physics and nuclear physics. The names 'tHooft and Van der Meer immediately jump to mind. Nuclear physicists at NIKHEF in Amsterdam helped gather evidence that nucleons are smaller outside the nucleus than when packed together with other nucleons inside it. However, recent work suggests that the effect may be less dramatic than was thought first.

Young Dutch postdocs in theoretical high energy physics seem to be swarming in such numbers all over the world that The Netherlands is apparently exporting not only flowers, bulbs, cheese and astronomers but also high energy physicists.

\section{Structure}

There is probably no other country with more research laboratories belonging to large companies per square kilometre than The Netherlands. In addition to hosting dozens of small industrial laboratories, it is the base for the research centres of commercial giants such as Philips, Shell, Unilever, DSM and AKZO. Several sizeable technological institutes, financed to some extent

\section{The Optical Piston}

Light induced drift can occur when a gas mixture (e.g. Na:Ar) is illuminated by a laser beam. If the laser is tuned to one of the Doppler wings of the $\mathrm{Na}$ absorption line, the $\mathrm{Na}$ atoms will move through the buffer gas as a result of velocity selective excitation combined with the state dependent collisional interaction between the $\mathrm{Na}$ and $\mathrm{Ar}$ atoms. A group at the University of Leiden led by $\mathrm{H}$. Woerdman has been working on this phenomenon and on a whole range of related light induced kinetic effects.

The optical piston is a dramatic manifestation of light induced drift. A mixture of $\mathrm{Na}$ and $\mathrm{Ar}$ vapour, homogeneously filling a capillary, is illuminated by a beam from a $\mathrm{CW}$ dye laser tuned in near resonance with a $\mathrm{Na}$ absorption line. The laser light pushes the $\mathrm{Na}$ atoms together into a steep front (the $\mathrm{Na}$ vapour is optically thick) which slowly travels through the capillary.

The figure gives a series of photos demonstrating the optical piston. The laser beam passes from left to right through a $15 \mathrm{~cm}$ long capillary containing the gas mixture. The bright spot in successive frames, starting at the top, taken every 30 seconds indicates the location of the high density $\mathrm{Na}$ front as it travels down the capillary. The part of the capillary that has been swept clear by the piston weakly fluoresces; the right-hand side of the capillary is dark since the laser light cannot penetrate into this region.

\section{E. Eliel, \\ Huygens Laboratory, University of Leiden}

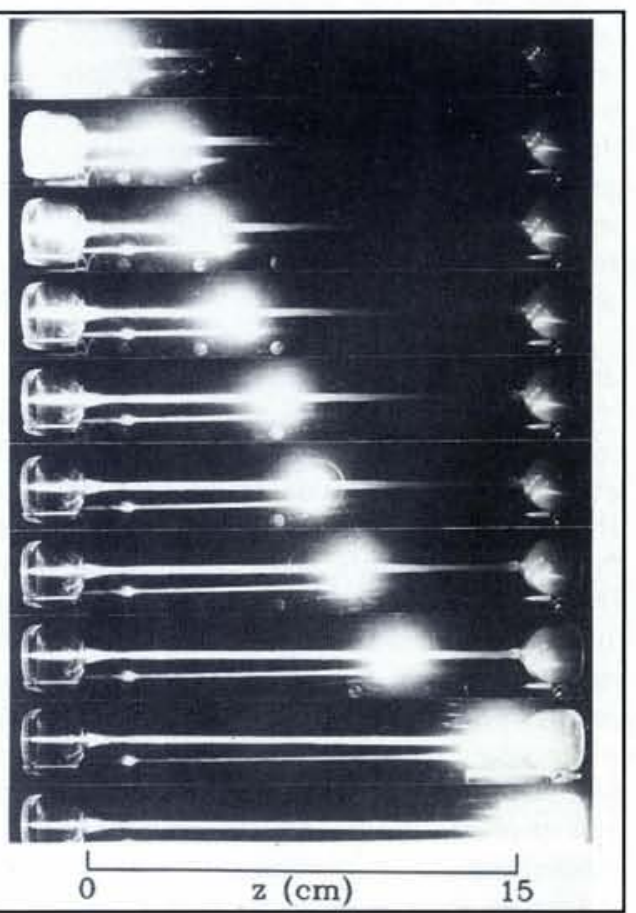




\section{Localization of Light}

Analogous to Anderson localization of electrons in disordered conductors, theory predicts that light waves can be localized in disordered media. A group headed by A. Lagendijk at the University of Amsterdam has been studying the propagation of light in disordered media for several years. Actual localization has not yet been demonstrated, but the graph shown here (taken from the thesis of M. van der Mark, 1990) gives evidence that Anderson localization has almost been reached.

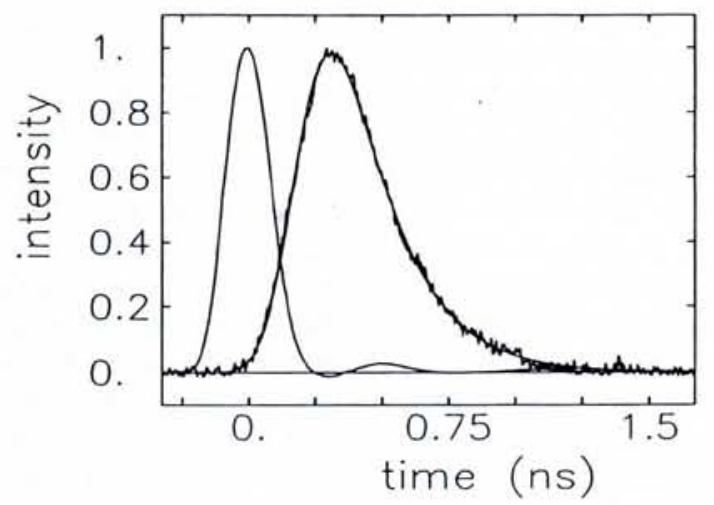

The graph records the results of a time-resolved experiment giving the transmission of 5 ps laser pulses at a wavelength in a vacuum of $600 \mathrm{~nm}$ through a $165 \pm 7$ $\mu \mathrm{m}$ thick slab of rutile $\mathrm{TiO}_{2}$ particles glued together with 1 weight $\%$ PMMA. The curve to the left is the measured response as a function of time of a diode to the pulses from the dye laser. The curves to the right are the measured (noisy) transmission curve and the fitted theoretical curve. The localization transition is expected to be given qualitatively by the loffe-Regel criterion, i.e. when the mean free path equals the wavelength divided by $2 \pi$. From the experimental value of the diffusion constant and the estimated refractive index one obtains a mean free path which is 1.86 times larger than the value given by the loffe-Regel criterion. This result provides strong evidence that the physicists in Amsterdam have nearly been able to obtain the Anderson localization of light.

\section{T. Nieuwenhuizen,}

by the government, also carry out physics research. Of these industrial laboratories and institutes, Philips and Shell employ by far the largest numbers of physicists (about 3000 all told). Private companies spent some 750 million guilders on physics research in The Netherlands in 1987. In the same year, government spending on physics research amounted to 270 million guilders so the bulk of physics research is going on in industry.

The industrial research is of course largely oriented towards R. and D. Philips concentrates heavily on semiconductors, materials and microelectronics. The main fields of interest for Shell are computational physics, hydrodynamics, catalysis and composites. There is clearly a wide gap between fundamental research and $R$. and $D$. The industrial giants therefore have relatively little influence on the educational and research systems in the universities. Nonetheless, academic and industrial researchers try to communicate extensively.

Just as two large companies domi-
Physics Laboratory, University of Amsterdam

\section{International Cooperation}

In a small country like the Netherlands the clever thing to do is to concentrate and coordinate physics research as it becomes more sophisticated, complex, international and inevitably more expensive. By founding FOM as long ago as 1946 , the Dutch physics community acquired an organization that could manage "big science" through its institutes. Meanwhile, the universities and FOM both carried out small science.

It was quite natural for FOM to become the Dutch partner in the EURATOM fusion research programme (through the FOM Institute for Plasma Physics, Rijnhuizen). NIKHEF in Amsterdam via FOM became the home base for participation in the field of high energy particle physics with CERN and in HERA at DESY in Germany. A joint French-Dutch project in nuclear physics, funded by FOM, involving the construction of the new AGOR cyclotron by $\mathrm{KVI}$, Groningen and In2P3, Orsay, is currently underway (see box on page 160: AGOR Takes Shape). FOM is also a founding member of JESSI and has participated in its planning. JESSI stands for the Joint European Sub-Micron Silicon Initiative, an industrial initiative that is supported by European Community and national programmes. Presently in an 18 month start-up phase, it is expected that the DIMES institute in Delft will play a major role in any Dutch research involving JESSI. NIKHEF in the meantime is building a storage ring called AmPS for its linear accelerator MEA. Another interesting development is the creation of the FELIX free electron laser facility at Rijnhuizen which will be used by scientists from a number of different fields based both in The Netherlands and elsewhere.

At a more personal level, university and FOM researchers work closely together in many national projects, take part in European Community programmes, and carry out experiments at the Daresbury synchrotron radiation facility in England and plan to do so at the ESRF in Grenoble, France. Indeed Dutch physicists consider it more-or-less routine to work with colleagues from all over the world. receive Ph.D.'s. The output of Dutch physicists in terms of the number of articles published has been rising over the years and now amounts to just under $2 \%$ of the world total. Measured by the citation rate, Dutch physicists rank seventh in the world. Roughly $31 \%$ of all articles with Dutch authors are written with colleagues working abroad, signalling that Dutch physics is extremely international in character.

\section{Planning}

One of the initial aims of FOM was to coordinate research at the national level and this led to a well-established system of peer review and national research planning. Planning and cooperation at the national level grew over the years. Areas of big science such as nuclear and high energy physics were 
of course the first. Fields such as materials and microelectronics have followed more recently.

Currents developments show a rapid build up of more and more dedicated institutes at universities. These institutes are not necessarily supported by FOM; nor are they shared exclusively by physicists. Formal cooperation between physicists and scientists from other disciplines at the national level can be identified in surface science and materials: for example, with chemists at the Debye Institute in Utrecht, at the Materials Study Centre in Groningen and in the FOM-ALMOS collaboration between FOM and the Universities of Amsterdam and Leiden. But there is also cooperation in mathematical physics (with mathematicians), computational physics (with computer scientists) and high resolution electron microscopy (with scientists from several disciplines at the recently established HREM Centre in Delft).

With encouragement from the government, the three technical universities have agreed on complementary research programmes in the fields of semiconductors and microelectronics and one outcome has been the formation of the Delft Institute for Microelectronics and Submicron Physics (DIMES). A

\section{Electron Wave Function Measured}

In 1988 scientists at AMOLF realized the dream of every atomic physicist. Using a laser belonging to the University of Amsterdam they actually measured the "classi$\mathrm{cal}^{\prime \prime}$ orbit time of the radial motion of a Rydberg electron around the atomic core. The key to the experiment was to excite the electron by a short laser pulse at the $n=19$ manifold of rubidium, thus forming a dispersion free wavepacket at time $t=0$. With a second pulse, the delay time of which can be adjusted, the atom is then ionized. Interaction with the laser field is only possible when the electron is close to the core. Peaks in the ionization yield occur if the delay is a multiple integral of the "classical" Bohr orbit time. This experiment directly demonstrates the link between classical and quantum mechanics.

The figure plots the observed probability of finding an electron close to the atomic core as a function of the time between the initial excitation laser pulse and the subsequent ionisation pulse. For this experiment, the complete $n=19$ manifold of rubidium was excited in a $645 \mathrm{~V} / \mathrm{cm}$ electric field. Successive peaks arise when the delay time is a multiple of the electron's orbit time around the nucleus.

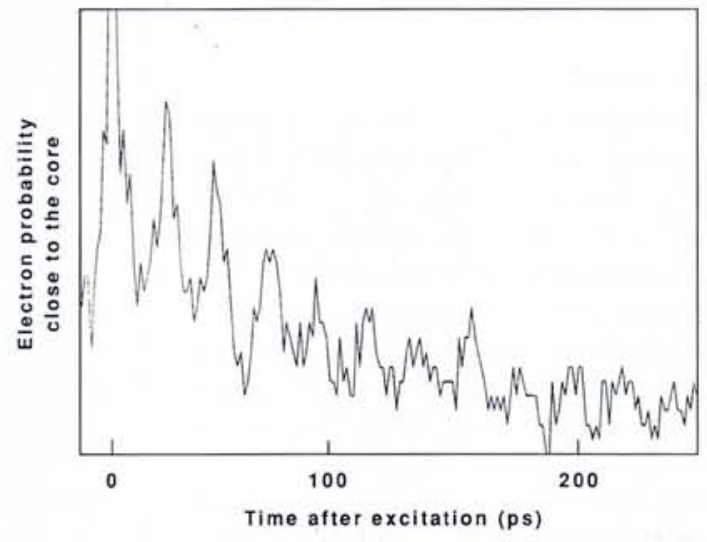

Institute for Atomic and Molecular Physics, Amsterdam

\section{Grenoble - Rhône-Alpes - France}

The European Synchrotron Radiation Facility is constructing a state of the art storage ring for $6 \mathrm{GeV}$ electrons and/or positrons to be operated as a high brilliance synchrotron radiation source in the field of $X$-ray from 1994 on. Financing of the ESRF is shared by 11 European countries.

\section{EUROPEAN SYNCHROTRON RADIATION FACILITY}

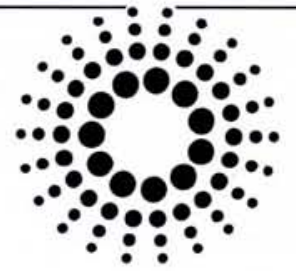

In the present build up phase, the European Synchrotron Radiation Facility offers you interesting career prospects and a unique opportunity to develop and apply your skills in an international hi-tech environment in fields as varied as

Materials Sciences: diffraction, elastic diffuse scattering, inelastic scattering, extended X-ray absorption fine structure.

condensed matter theory, magnetic scattering.

X-ray Beam Lines: design, imaging, optics, detectors.

Physics,

Mechanics,

Ultra High Vacuum,

Power Supplies,

Applied Computer Science: hardware \& Software

Chemistry,

Magnets: undulators $\mathcal{E}$ wigglers

Hydraulics and Cooling.

Radio frequency.

Electronics,

Remote Control \& Operation of Complex Machines. Biology.

We recruit now:

\section{Scientists*, Engineers and Technicians}

*Scientists are generally on fixed-term contracts (max. 5 years)

who give evidence of relevant education, and who have experience in research institutes, universities or high-tech industries. Candidates with at least some years of experience in a similar situation, or with a professional background combining education and/or experience from one or more of the fields mentioned above are particularly invited to apply
The working language is English:- knowledge of French is desirable.

For more information about the ESRF, employment conditions and specific vacancies, you can give your name, address and field of professional experience directly to us in Grenoble, and we shall provide you with the information needed. and an "Application Form".

The completed "Application Form" must reach us at thellatest on 15 September 1990.

ESRF (Recruitment Officer), Boîte Postale 220, F-38043 Grenoble Cedex.

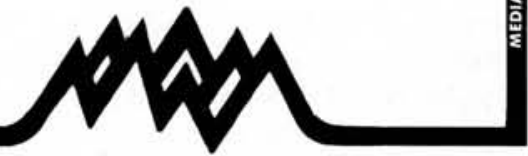


major installation in the Institute is an electron beam pattern generator.

Two facilities, in Amsterdam and Nijmegen, for high magnetic fields also coordinate their activities across a broad front. Successful national research programmes exist in the fields of integrated circuit technology, optoelectronics and high temperature superconductors. In addition to the FELIX free electron facility, a smaller and more modest activity in the area has recently been launched by the technical universities of Enschede and Eindhoven.

\section{Employment}

Graduates with both first degrees and doctorates encounter little difficulty in finding positions. Over the years, the number of young physicists has just about kept pace with the demand. The relatively constant requirements of the large multinational companies and the traditional ease with which the Dutch go abroad are important factors in creating this stable situation.

However, during the last few years the situation in the "education" market has rapidly deteriorated. University courses in teacher training are right now almost empty. A real problem is in the making. The image of the teaching profession is poor, mainly because of low starting salaries for graduates and permanent unrest in the education system owing to difficulties with the budget. The government claims to be aware of the disturbing situation but still does not provide adequate funding to do something about it.

\section{The Physical Society}

The Netherlands has a single physical society called The Netherlands' Physical Society (or Nederlandse Natuurkundige Vereniging) founded in 1921 by Lorentz, Zeeman, Kamerlingh Onnes and others. Of the 8000 or so working physicists in the Netherlands, about 3500 are members. The main activities of the Society involve promoting the interests of the Dutch physics community through contacts with national and international societies and with university and government officials, organizing a general Spring Meeting and meetings of the Society's 16 divisions and sections, publishing two periodicals and maintaining two chairs (at the Universities of Amsterdam and Delft).

The overall image of physics in The Netherlands is that of an internationally oriented, productive, healthy and enthusiastic community - features that everybody hopes to have shown you in Amsterdam this month.

\section{AGOR Takes Shape}

AGOR, the Accelerator Groningen - Orsay, as the acronym suggests is a joint Dutch-French project to build a superconducting cyclotron with the unique capability to accelerate both protons (to $100-200 \mathrm{MeV}$ ) and heavy ions up to uranium (to 6-100 MeV/nucleon). Representing the largest ever collaboration of its type, the machine is being built at IPN, the Institute of Nuclear Physics, Orsay in France. It will be installed at KVI, the Nuclear Accelerator Institute in Groningen, The Netherlands where it will be used by physicists from both countries. The superconducting magnet coils and their cryogenic systems are presently being fabricated ready for field mapping starting in mid-1991, and the first beam tests at Orsay in 1993.

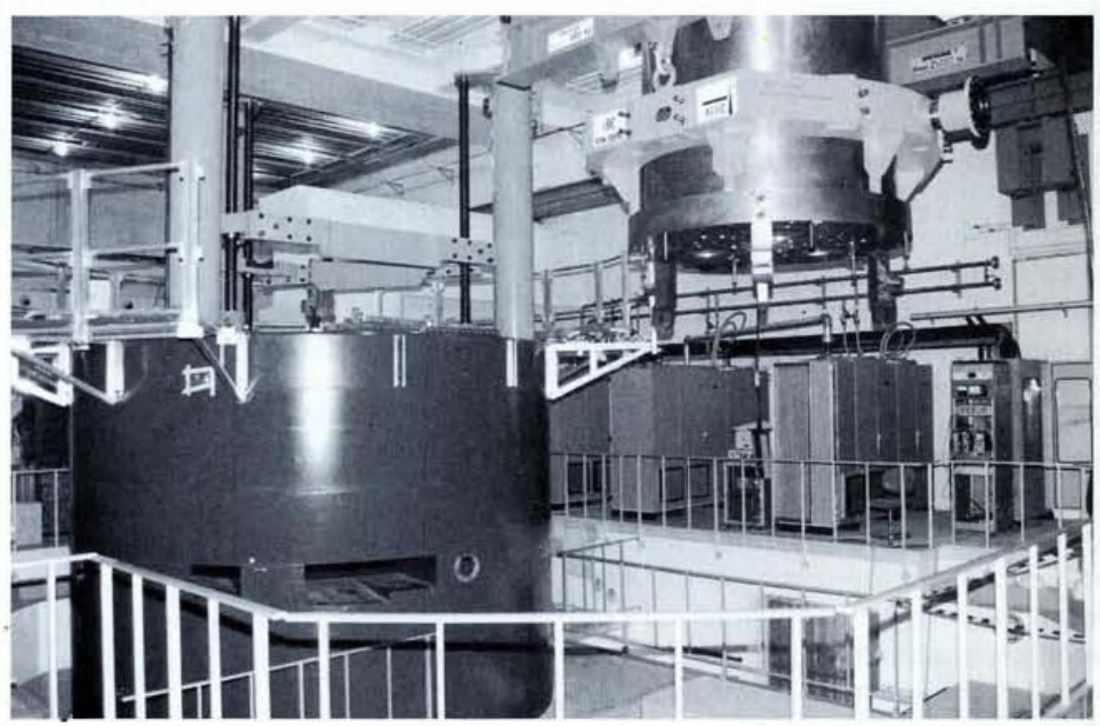

The photo shows the AGOR cyclotron under construction at Orsay. To the right is one of the two pole pieces of the superconducting magnet structure. It is $1.9 \mathrm{~m}$ in diameter and has three cylindrical spaces for the RF resonators separating three magnetic sectors. Not visible is the complicated form of the pole face which has been optimised for the production of the very steep radial field gradient that is required to allow protons as well as other ions to be accelerated in a stable configuration. A pair of superconducting coils, $2.4 \mathrm{~m}$ in diameter, weighing 25 tonnes and wound using $\mathrm{NbTi}$ conductor, will be placed around each of the pole pieces that are separated at the median plane by a gap of $70 \mathrm{~mm}$ between opposing magnet sectors.

To the left is shown the $4.4 \mathrm{~m}$ diameter cylindrical iron yoke which will encircle the two sets of pole/coil assemblies. Rectangular and square apertures around the median plane are for the beam exit (dummy exits are not visible) and for inserting instrumentation. The $200 \mathrm{~mm}$ diameter hole is for one of the three radial tie rods that support the two pole/coil assemblies; other tie rods pass vertically.

Racks of cabinets for RF power supplies for the resonators that work at the relatively high frequency of $62 \mathrm{MHz}$ (40 MHz is more usual) are found in the background.

H.E. Schreuder, KVI, Groningen

\section{Balances in the Atmosphere and the Energy Problem Editor: E.W.A. Lingeman \\ Proceedings of the 59th WE-Heraeus Seminar Bad Honnef, FRG / 4-7 February 1990}

Organized in co-operation with the EPS Action Committee on Physics and Society

A. Tartaglia: Induced Critical Conditions H. Grassl: The Greenhouse Effect K.Ya. Vinnikov: Anthropogenic Change A.P.M. Baede: Greenhouse Impact R. Kümmel: Removal, Disposal of $\mathrm{CO}_{2}$ D.O. Hall: $\mathrm{CO}_{2}$ Cycling

\section{K. Heinloth: Summary}

Orders in writing to EPS Secretariat, Geneva with payment (cheques drawn on a Swiss bank) or transfer:

Société de Banque Suisse, CP, CH-1211 Geneva 11, Acc. No.: 164.899

Swiss Post Office, Acc. No.: CCP 12-19107-4
S.G. Zvenigorodsky: Numerical Simulations A.S. Silbergleit: Thermodynamics A.K. Schatz: Nuclear Energy W. Turkenburg: Photovoltaic Energy C. Marchetti: Energy Without $\mathrm{CO}_{2}$ G. Benedek: The EPS and UNESCO

PRICE: IOM's - $60 \mathrm{Sfr}$ other - $120 \mathrm{Sfr}$ 